\title{
EXTREME ASYMMETRY AND CHIRALITY A CHALLENGING QUANTIFICATION
}

\author{
Michel Petitjean ${ }^{1,2}$
}

${ }^{1}$ Université de Paris, BFA, CNRS UMR 8251, INSERM ERL U1133, F-75013 Paris, France.

2 E-pôle de Génoinformatique, CNRS UMR 7592, Institut Jacques Monod, 75013 Paris, France.

E-mail: petitjean.chiral@gmail.com,michel.petitjean@univ-paris-diderot.fr. http://petitjeanmichel.free.fr/itoweb.petitjean.html. ORCID: 0000-0002-1745-5402.

\begin{abstract}
Symmetry operators must be defined relatively to a space and to a metric. Complex symmetries are typically considered in the case of the cartesian product of the Euclidean space by a space whose elements are tuples of colors. While interesting open problems exist in this field, challenging ones remain in the Euclidean case, without invoking colors. We present some of these problems in the case of extreme chirality figures and of extreme asymmetrical probability distributions.
\end{abstract}

Keywords: chirality, mirror symmetry, asymmetry, skewness.

MSC 2020: 51M16, 52A40, 60E15

\section{INTRODUCTION}

It is crucial to understand that mathematical constructs are models of real physical situations, and that a mathematical model of symmetry is a simplified image in our mind of some physical situation in which we would like to see symmetry. This concept of symmetry gives rise to an unifying mathematical definition working in most pratical situations (Petitjean, 2007). It involves first an unambiguous definition of the space in which symmetries operators must be defined. Then, it involves the choice of a metric, because symmetry operators must be distance-preserving. The axioms defining a metric (see Deza and Deza, 2009), are not used to build the unifying definition of symmetry. In other words, the metric is not required to be a true one, e.g. it is not required to be positive definite. This is very useful for physics applications in the relativistic spacetime (Petitjean, 2019, 2020a). However, there must be no ambiguity about the choice of the metric. When this latter is not clearly specified, incoherent or contradictory conclusions occur, thus generating controversies (Petitjean, 2020b). 
Then, a mathematical definition of chirality is needed. The main one, due to Lord Kelvin (1894, 1904), is highly used for teaching, particularly in chemistry and in biology. It targets the Euclidean space, but it is not a mathematical definition. An other definition exists, which is sometimes used in high energy physics. It is based on the geometric product (Hestenes and Sobczyk, 1987), but it is restricted to quadratic spaces. It is why we retain here the general definition of Petitjean (2010, 2020c), which, in the case of Euclidean spaces, recovers the one of Kelvin and the one based on the geometric product. Our main purpose here is to measure the deviation from achirality, i.e., given an idealized chiral object, measure how much it is chiral rather than simply deciding that it is chiral or achiral. Despite that some confusion still sometimes occurs about the role of mirrors in the Euclidean spaces, as noticed by Petitjean (2020d), in the next section we focus on the mathematical aspects of chirality measures rather than the basic concepts of mirror symmetry.

\section{METHODS}

\subsection{A simple test set}

Since the end of the $19^{t h}$ century, many methods were developed to build quantitative measures of the degree of symmetry and of the degree of chirality of various objects: see Petitjean (2003) for a review. Such measures depend only on the objects themselves (geometric figures, molecules, probability distributions, etc.), and by no way they should be confused with any kind of light-matter interaction. It means that a chiral object, for example a scalene triangle in the plane, is declared to be chiral while no light-matter interaction is invoked. Before looking at complex situations, it is enlighting to consider the simplest possible non trivial case of asymmetry: three points on the real line. The shape of this three points set depends on only one parameter, the ratio $\alpha$ of the lengths of the two adjacent segments. For this set, no rotation is possible except the identity, and so only translations and mirror reflections are considered. According to Petitjean (2013), in this situation a safe chirality measure $\chi$ should have the following properties:

1. $\chi$ is function of only the unique parameter of the set, $\alpha$.

2. $\chi$ is a continuous function of $\alpha$.

3. $\chi(1)=0$ (the set is achiral when $\alpha=1$ ).

4. $\chi(\alpha)=0 \Longrightarrow \alpha=1$.

5. $\chi(\alpha)=\chi(1 / \alpha)$

Property 1 means that $\chi$ is insensitive to isometries and scaling, because the shape parameter $\alpha$ is insensitive to isometries and scaling (thus, $\chi$ is location independant). 
Property 2 is required to preclude ambiguities in the meaning of the measure in the neighborhood of the points of dicontinuity. Properties 3 and 4 mean that $\chi$ takes a null value if and only if the set is achiral. This is not the case for many asymmetry coefficients known in statistics, such as the skewness (see section 4.2.2 in Petitjean, 2003). Property 5 means that $\alpha$ and $1 / \alpha$ correspond to similar sets (see Property 1 ).

These five properties, enumerated above, are satisfied by the chiral index of Petitjean (2002), which is presented in section 2.3, and that we also denote $\chi$ further in the text. For the three points set, the chiral index is given in equation 1 (Petitjean, 2013).

$$
\chi=\frac{(1-\alpha)^{2}}{4\left(1+\alpha+\alpha^{2}\right)}
$$

In equation $1, \chi$ takes values in $[0 ; 1 / 4]$, the value $1 / 4$ being reached for $\alpha=0 . \chi$ is insensitive to isometries and scaling. It happens that, except for the chiral index, many chirality measures proposed in the literature fail to satisfy one or several of the five required properties, despite that they are supposed to work for sets more complex than the three points one (Petitjean, 2003). If we except the asymmetry measures developed by the statisticians (see section 4.2 in Petitjean, 2003), which are intended for symmetry testing rather than to measure asymmetry, this is rather disappointing.

\subsection{Direct symmetry}

A general procedure was proposed to build chirality and symmetry measures, based on the existence of a metric in the space of objects (see section 6 in Petitjean, 2003), but it was mentioned that, in the case of direct symmetry, it is restricted to finite cardinality sets. This metric must not be confused with the one used in the unifying definition of symmetry mentioned in section 1. It is recalled that an object is a function having its input argument in a metric space. Thus, the metric in the space of objects (the output of the function) should not be confused with the metric in the space of the input argument of the function.

An open problem is to quantify direct symmetry, discarding any reference to a specified type of direct symmetry, in the case of infinite cardinality sets. For finite cardinality sets, with or without colors, a direct symmetry index (DSI) was defined (Petitjean, 1999a). As stated in section 2.1, apart the identity no rotation exists in the case of the real line. Thus, the simplest non trivial case is the one of three points in the plane, and we call this set a triangle. This triangle may also be viewed as the distribution of three equiprobable points in the plane. It was shown that the most direct symmetric triangle has its angles equal to $\pi / 4, \pi / 8$ and $5 \pi / 8$ (Petitjean, 1999a). Exhibiting extreme direct symmetric figures for other classes of finite sets remains to do. 


\subsection{The chiral index}

The chiral index $\chi$, introduced for finite colored sets by Petitjean (1997), was first applied to a mathematical model of molecular conformers (Petitjean, 1999b), which led further to identify an extreme chirality conformer, the [6.6]chiralane (Schwartz and Petitjean, 2008). Then it was extended to weighted sets (Petitjean, 2001), and finally it was generalized to probability distributions (Petitjean, 2002). The general case takes in account color symmetry, either through an additional graph structure (Petitjean, 1999b), or trough a class of probability distributions involving both a space of colors and an Euclidean space (Petitjean, 2002). The resulting so-called color mixtures model has a formal relation with the Wasserstein metric (Dobrushin, 1970; Rachev, 1991), encountered in the MongeKantorovitch transportation problem (Rachev and Rüschendorf, 1998). It is related to Procrustes methods (Petitjean, 2002), which are used in shape analysis and psychometrics (ten Berge, 1977, 2006), and it received applications in docking theory (Petitjean, 2004).

In the non-colored case, a random vector $X$ taking values in $\mathbb{R}^{d}, d \geq 1$, is considered, and $\chi$ is defined in equation 2 , where the quote denotes a vector transposition:

$$
\chi=\frac{d}{4 T} \operatorname{In} f_{\{W, R, t\}} E(X-(R Y+t))^{\prime}(X-(R Y+t))
$$

Where:

$T$ is the inertia of $X$ (i.e. the trace of its covariance matrix), assumed to be finite and non null.

$Y$ is a random vector distributed as a mirror image of $X$.

$W$ is a joint distribution of the couple $(X, Y)$.

$R$ is a rotation of $\mathbb{R}^{d}$.

$t$ a translation of $\mathbb{R}^{d}$.

The infimum of the expectation is its lower bound, taken over the set of all rotations and translations applied to $Y$, and over all joint distributions of $(X, Y)$. It is also the $L^{2}$-Wasserstein distance between the distribution of $X$ and the one of its mirror image $Y$, minimized for all direct isometries applied to $Y$. In the case of color symmetry, not all joint distributions are allowed, because there are constraints due either to an additional graph structure or to the space of colors (Petitjean, 1999b, 2002). The full theory is outside the scope of the present paper, and only the non-colored case is considered here.

The chiral index is a parameter of the distribution of $X$. It takes values in $[0 ; 1]$ and it is insensitive to isometries and scaling. It is null if and only if the distribution is achiral, i.e. if it has an indirect symmetry. It follows that $\chi$ is an asymmetry coefficient of the distribution of $X$. 
The optimal translation is $t=0$ when $X$ is centered, i.e. when $E X=0$. Specific expressions are known for the optimal rotation when $d=2$ and $d=3$ (Petitjean, 2002). In the case of a finite number of equiprobable points, an expression of the optimal rotation valid for any $d$ value was proved to stand (ten Berge, 2006).

In the case of a discrete distribution of $n$ equiprobable values, or in the case of the empirical distribution of a sample of $n$ observations in $\mathbb{R}^{d}$, equation 2 can be rewritten as follows (Petitjean, 1997, 2002):

$$
\left.\chi=\frac{d}{4 T} \operatorname{Min}_{\{P, R\}} \operatorname{Tr}\left(X-P Y R^{\prime}\right)\left(X-P Y R^{\prime}\right)^{\prime}\right)
$$

Where:

$X$ is the $n$ by $d$ matrix containing the centered observations

(i.e., the $n$ observations are translated to get a null mean).

$Y$ is a matrix of $n$ lines and $d$ columns which is a mirror image of $X$.

$P$ is a $n$ by $n$ permutation matrix.

$R$ is a rotation of $\mathbb{R}^{d}$.

$\operatorname{Tr}$ is the trace operator.

$T=\operatorname{Tr}\left(X^{\prime} X\right)$, is the inertia of $X$; it is assumed to be non null.

When $d=1, R$ is the identity, and equation 3 receives a simple expression because the optimal permutation is known: it is such that the sample ordered in increasing values is correlated with the same sample (no sign change), ordered in decreasing values (see Lemma 2 in Petitjean, 1997, and equations 2.9.3-2.9.5 in Petitjean, 2003). Denoting by $r_{m}$ the corresponding correlation coefficient, we have:

$$
\chi=\left(1+r_{m}\right) / 2
$$

\section{RESULTS AND DISCUSSION}

\subsection{Some maximally chiral sets}

The chiral index cannot reach its upper bound $\chi=1$ unless the covariance matrix of $X$ is proportional to the identity (Petitjean, 2002). This condition stands both in the colored case and in the non-colored case. It is also a sufficient condition in the case of $n$ equiprobable colored points in $\mathbb{R}^{d}$, not two of them having the same color (Petitjean, 2002). This condition is filled for the regular simplex (Petitjean, 1997), for the $d$-cube and its dual, and for the other regular polytopes for which there is no preferred principal direction (Petitjean, 2002). All these geometric figures are highly symmetric when the colors are discarded. 
However, extreme chirality figures are more difficult to exhibit in the non-colored case. The most chiral three points set in the real line is got for $\alpha=0$, which means that two of the three points lie at the same location, and thus $\chi=1 / 4$ (see equation 1 ). The most chiral three points set in the plane is got for a triangle for which the ratios of its squared sides lengths are $1:(4+\sqrt{15}):(5+\sqrt{15}) / 2$ (Petitjean, 1997), and for which $\chi=1-2 \sqrt{5} / 5$ $(\chi \approx 0.105573)$. This triangle and other maximally asymmetric triangles (with colored vertices), are displayed in two open access documents: see Bouissou and Petitjean (2018) and Petitjean (2018).

The most chiral triangle (with non colored vertices), was proposed as a candidate for the "most scalene triangle", but it is an obtuse triangle. For the "most scalene acute triangle", it was proposed by Petitjean (2015) and then by Bouissou and Petitjean (2018), to retain the triangular face of the most chiral disphenoid (Petitjean, 2015), which is known to be an acute triangle. The chiral index of this disphenoid is $\chi=3(13-6 \sqrt{2}) / 97$ $(\chi \approx 0.139630)$. The ratios of the squared sides lengths of its triangular face are 1 : $3-\sqrt{2} / 2: 3$ (Petitjean, 2015). However, the planar coordinates of this triangular face, given by Bouissou and Petitjean (2018), have a more complicated expression than its three-dimensional coordinates.

\subsection{Seeking for maximally chiral probability distributions}

Until now, we considered maximally chiral figures within specific classes of finites sets of points. Finite sets of equiprobable points (without colors) are of interest because they can be viewed as random samples from some parent probability distribution. Due to a convergence theorem (Petitjean, 2002), the sample chiral index is a statistic which is an estimator of the chiral index of the parent distribution. It is why it was proposed for symmetry testing: see Monte-Carlo experiments in Petitjean (2020e). Finding closed forms for the asymptotic distribution of this estimator is an open problem.

In the univariate case $(d=1)$, the upper bound of the chiral index is known to be $1 / 2$. It is asymptotically reached for the Bernouilli distribution of parameter $p$ tending to 0 or to 1 (Petitjean, 2002). In the multivariate case $(d>1)$, the upper bound of the chiral index is known to be in $[1 / 2 ; 1]$ (Petitjean, 2008). This interval was refined to [ $1-1 / \pi ; 1-1 / 2 \pi]$ in the case $d=2$, with a conjecture that the upper bound is $1-1 / \pi$, this bound being asymptotically reached by a family of bivariate distributions which was exhibited (Coppersmith and Petitjean, 2005). This family of bivariate distributions is presented below in the complex plane. 
Fix an arbitrarily small positive real $\epsilon>0$ and choose an even integer $m>1 / \epsilon$. Then set $\omega=e^{i \frac{2 \pi}{2 m}}$, where $i$ is the pure imaginary number (thus, $\omega^{2 m}=1$ ). Select an integer $r>m^{4} / \epsilon^{2}$, and select an even integer $k>\frac{r^{m-1}}{\epsilon}$. We define $S=\sum_{j=0}^{m-1} \omega^{j} r^{\frac{j}{2}}$, and we define a complex vector $z \in \mathbb{C}^{n}$, with $n=1+r+r^{2}+\ldots+r^{m-1}+k+\frac{k}{2}+\frac{k}{2}$, which means that $z$ has $m+3$ blocks of elements, shown in Table 1 .

The $n$ complex components of $z$ define a set $n$ points in the complex plane, which have a null sum. The points within a common block share a common location, which means that in fact there are $m+3$ distinct points, each one being weighted by its multiplicity. That can be seen as a discrete bivariate probability distribution in the plane, whose support is located over $m+3$ points, and to each of these $m+3$ points is associated a probability equal to its relative multiplicity. The expectation is null and the variance matrix is proportional to the identity because $z^{\prime} z=0$.

\begin{tabular}{|c|c|c|}
\hline block & component $z_{j}$ & multiplicity \\
\hline 0 & 1 & 1 \\
\hline 1 & $\omega / r^{\frac{1}{2}}$ & $r$ \\
\hline 2 & $\omega^{2} / r$ & $r^{2}$ \\
\hline$\vdots$ & $\vdots$ & $\vdots$ \\
\hline$j$ & $\omega^{j} / r^{\frac{j}{2}}$ & $r^{j}$ \\
\hline$\vdots$ & $\vdots$ & 领 \\
\hline$m-1$ & $\omega^{m-1} / r^{\frac{m-1}{2}}$ & $r^{m-1}$ \\
\hline$m$ & $-S / k$ & $k$ \\
\hline$m+1$ & $i S / k$ & $k / 2$ \\
\hline$m+2$ & $-i S / k$ & $k / 2$ \\
\hline
\end{tabular}

Table 1: The locations of the $m+3$ elements of $z$, with their multiplicities.

To summarize, the family of sets presented in Table 1 has four parameters: the real $\epsilon>0$, and the integer numbers $m>1 / \epsilon, r>m^{4} / \epsilon^{2}$, and $k>\left(r^{m-1}\right) / \epsilon$. The value of $\epsilon$ must be arbitrarily small to approach the limiting value of the chiral index, equal to $1-1 / \pi$, conjectured to be the upper bound for bivariate probability distributions. Thus, the three integer parameters increase to infinity when $\epsilon$ tends to zero. When $\epsilon$ decreases, visualizing graphically the geometric figures generated by the $n$ points of $z$ becomes rapidly very difficult, not to say practically impossible. 


\section{CONCLUSIONS}

In the literature, few publications are devoted to the identification of maximally asymmetric probability distributions or sets, with or without colors, both for direct and indirect symmetry. Computing the chiral index is an efficient way to tackle this problem.

In the non-colored case, the main open problems are that the upper bound of the chiral index is unknown for multivariate distributions $(d \geq 2)$, and that families of distributions approaching this upper bound remain to be exhibited. The conjecture of Coppersmith and Petitjean (2005), about the upper bound for $d=2$, remains to be proved or disproved.

An other open problem is, starting from a given chiral distribution, define what could be its closest achiral image, if existing. Some preliminary results exist (Petitjean, 2006), but this problem is far to being solved.

\section{REFERENCES}

Bouissou, C., Petitjean, M. (2018) Asymmetric Exchanges, J. Interd. Method. Iss. Sci., 4, 1-18, https://hal.archives-ouvertes.fr/hal-01782438.

Coppersmith, D., Petitjean, M. (2005) About the Optimal Density Associated to the Chiral Index of a Sample from a Bivariate Distribution, Compt. Rend. Acad. Sci. Paris, Sér. I, 340(8), 599-604, https://doi.org/10.1016/j.crma.2005.03.011.

Deza, M.M., Deza,E. (2009) Encyclopedia of Distances, Springer, Berlin, https://doi.org/10.1007/978-3-642-00234-2.

Dobrushin, R.L. (1970) Prescribing a system of random variables by conditional distributions, Theor. Prob. Appl. 15(3), 458-486, https://doi.org/10.1137/1115049.

Hestenes, D., Sobczyk, G. (1987) Clifford Algebra to Geometric Calculus, A Unified Language for Mathematics and Physics, section 3.8, D. Reidel Publising Co, Dordreht, The Netherlands, https://doi.org/10.1007/978-94-009-6292-7.

Lord Kelvin (1894) The Molecular Tactics of a Crystal, sect. 22, footnote p. 27, Clarendon Press, Oxford, UK, https://archive.org/details/moleculartactics00kelviala.

Lord Kelvin (1904) Baltimore Lectures on Molecular Dynamics and the Wave Theory of Light, appendix H, sect. 22, footnote p. 619, C.J. Clay and Sons, Cambridge University Press Warehouse, London, UK, https://archive.org/details/baltimorelecture00kelviala.

Petitjean, M. (1997) About Second Kind Continuous Chirality Measures. 1. Planar Sets, J. Math. Chem., 22(2-4), 185-201, https://doi.org/10.1023/A:1019132116175.

Petitjean, M. (1999a) Calcul de chiralité quantitative par la méthode des moindres carrés (in French), Compt. Rend. Acad. Sci. Paris, Sér. IIc, 2(1), 25-28, https://doi.org/10.1016/S1387-1609(99)80034-6.

Petitjean, M. (1999b) On the Root Mean Square Quantitative Chirality and Quantitative Symmetry Measures, J. Math. Phys., 40(9), 4587-4595, https://doi.org/10.1063/1.532988.

Petitjean, M. (2001) Chiralité quantitative: le modèle des moindres carrés pondérés (in French), Compt. Rend. Acad. Sci. Paris, Sér. IIc, 4(5), 331-333, https://doi.org/10.1016/S1387-1609(01)01241-5.

Petitjean, M. (2002) Chiral mixtures, J. Math. Phys., 43(8), 4147-4157, https://doi.org/10.1063/1.1484559.

Petitjean, M. (2003) Chirality and symmetry measures: a transdisciplinary review, Entropy, 5(3), 271-312, https://doi.org/10.3390/e5030271. 
Petitjean, M. (2004) From shape similarity to shape complementarity: toward a docking dheory, J. Math. Chem. 35(3), 147-158, https://doi.org/10.1023/B:JOMC.0000033252.59423.6b.

Petitjean, M. (2006) À propos de la référence achirale (in French), Compt. Rend. Chim., 9(10), 1249-1251, https://doi.org/10.1016/j.crci.2006.03.003.

Petitjean, M. (2007) A definition of symmetry, Symm. Cult. Sci., 18(2-3), 99-119, https://hal.archives-ouvertes.fr/hal-01552499.

Petitjean, M. (2008) About the upper bound of the chiral index of multivariate distributions, AIP Conf. Proc., 1073, 61-66, https://doi.org/10.1063/1.3039023.

Petitjean, M. (2010) Chirality in metric spaces, Symm. Cult. Sci., 21(1-3), 27-36, http://journal-scs.symmetry.hu/issue-content/?volume=21\&issue=1-3.

Petitjean, M. (2013) The chiral index: Applications to multivariate distributions and to 3D molecular graphs. Proceedings of the SOR'13 Conference, Dolenjske Toplice, Slovenia, September 25-27, pp. 11-16, https://hal.archives-ouvertes.fr/hal-01952400.

Petitjean, M. (2015) The most Chiral Disphenoid, MATCH Commun. Math. Comput. Chem., 73(2), 375-384, http://match.pmf.kg.ac.rs/content73n2.htm.

Petitjean, M. (2018) Defining and measuring asymmetry, First European Asymmetry Symposium, https://hal.archives-ouvertes.fr/hal-01778387.

Petitjean, M. (2019) About chirality in Minkowski spacetime, Symmetry, 11(10), 1320, https://doi.org/10.3390/sym11101320.

Petitjean, M. (2020a) Chirality of Dirac spinors revisited, Symmetry, 12(4), 616, https://doi.org/10.3390/sym12040616.

Petitjean M. (2020b) Molecular chirality in classical spacetime: solving the controversy about the spinning cone model of rotating molecules, Chem. Eur. J., 26(47), 10648-10652, https://doi.org/10.1002/chem.201904247.

Petitjean, M. (2020c) Chirality in metric spaces. In memoriam Michel Deza, Optim. Lett., 14(2), 329-338, https://doi.org/10.1007/s11590-017-1189-7.

Petitjean, M. (2020d) Comment on "Bad language": Resolving some ambiguities about chirality, Angew. Chem. Int. Ed., 59(20), 7650-7651, https://doi.org/10.1002/anie.201904314.

Petitjean, M. (2020e) Tables of quantiles of the distribution of the empirical chiral index in the case of the uniform law and in the case of the normal law, arXiv:2005.09960 [stat.ME], https://arxiv.org/abs/2005.09960.

Rachev, S.T. (1991) Probability Metrics and the Stability of Stochastic Models, Wiley, New York, Chap. 6.

Rachev, S.T., Rüschendorf, L. (1998) Mass Transportation Problems, Springer, New York, Vol. I, Chap. 1, https://doi.org/10.1007/b98893.

Schwartz, A., Petitjean, M. (2008) [6.6]Chiralane: A Remarkably Symmetric Chiral Molecule, Symmetry Cult. Sci., 19(4), 307-316, https://hal.archives-ouvertes.fr/hal-01941526.

ten Berge, J.M.F. (1977) Orthogonal Procrustes rotation for two or more matrices, Psychometrika 42(2), 267-276, https://doi.org/10.1007/BF02294053.

ten Berge, J.M.F. (2006) The rigid orthogonal Procrustes rotation problem, Psychometrika 71(1), 201-205, https://doi.org/10.1007/S11336-004-1160-5. 